\title{
COSTEP/SOHO observations of energetic electrons far upstream of the Earth's bow-shock
}

\author{
A. Klassen, R. Gómez-Herrero, E. Böhm, R. Müller-Mellin, B. Heber, and R. Wimmer-Schweingruber \\ Institut für Experimentelle und Angewandte Physik, Universität Kiel, 24118 Kiel, Germany
}

Received: 5 July 2007 - Revised: 14 January 2008 - Accepted: 4 March 2008 - Published: 13 May 2008

\begin{abstract}
We have analyzed 124 electron bursts at energies above $0.25 \mathrm{MeV}$ observed with the EPHIN/COSTEP instrument onboard the SOHO spacecraft far upstream of the Earth's bow-shock at the libration point L1 from 1996 through 2005. Most of the bursts were observed during low solar activity (in 1996-1997 and in 2005) and all 124 bursts were not associated with solar particle events. It is shown that some upstream events are detected at energies above $0.7 \mathrm{MeV}$.

We find that the event occurrence number shows a distinct seasonal variation with maxima around equinoxes and minima near solstices. This together with a close correspondence between the event occurrence number with maxima in solar wind speed $\left(V_{s w}\right)$, geomagnetic activity index $\left(A_{p}\right)$ and in the southward interplanetary magnetic field (IMF) component $\left(B_{z}\right)$ indicates that the observed events can be explained in terms of leakage of magnetospheric particles during enhanced geoactivity rather than by acceleration at the Earth's bow-shock.
\end{abstract}

Keywords. Interplanetary physics (Energetic particles; Interplanetary magnetic fields; Planetary bow shocks)

\section{Introduction}

A presence of energetic ions $(<1-2 \mathrm{MeV})$ upstream of the Earth's bow-shock accelerated inside the magnetosphere and/or at the bow-shock is well known since the 1960s (e.g. Fan et al., 1964; Asbridge et al., 1968). Most of such upstream bursts were observed near the bow-shock at $\sim 25 R_{E}$, where $R_{E}$ - Earth's radius (e.g. Krimigis et al., 1978), but also far upstream from the bow-shock at the libration point (L1) at $\sim 240 R_{E}$ (Balogh et al., 1978; Scholer et al., 1981).

Correspondence to: A. Klassen

(klassen@physik.uni-kiel.de)
The upstream ion bursts often associated with energetic electrons $(>30 \mathrm{keV})$ were explained in two ways: (1) the leakage of magnetospheric particles accelerated within the magnetosphere (e.g. Sarris et al., 1978; Krimigis et al., 1978), (2) the acceleration by the bow-shock (e.g. Scholer et al., 1981, and references therein). Scholer et al. (1981) distinguish two groups of upstream events: the first group of proton bursts is accompanied by energetic electrons $(\geq 75 \mathrm{keV})$, and the second group is unaccompanied by energetic electrons. It is suggested that group 1 is of magnetospheric origin, while group 2 is bow-shock associated.

Sanderson et al. (1981) investigated the orientation of the interplanetary magnetic field (IMF) at L1 and found that upstream events occur mainly during times of radial IMF, i.e. when the connection to the magnetosphere is optimal (see also Desai et al., 2000). On the other hand, recent multispacecraft investigations (Haggerty et al., 2000) show that $>40 \%$ of ion $(>15 \mathrm{keV})$ upstream events are observed during times of nonradial IMF orientation.

Observation of energetic $(<30 \mathrm{keV})$ upstream electrons were first reported by Fan et al. (1964) and were later studied in more detail by Anderson (1981, and references therein). It was suggested that these electrons are accelerated near to the shock surface. On the other hand Krimigis et al. (1978) reported observations of $>220 \mathrm{keV}$ electrons upstream of the bow-shock and suggested that their source lies within the magnetosphere. Formisano (1979) found that electrons $>43 \mathrm{keV}$ originate in the exterior cusp. Bieber and Stone (1982) also provided strong evidence that the high energy component $(\geq 200 \mathrm{keV})$ of upstream electrons originates in the inner magnetosheath or magnetosphere.

Previous statistical studies showed that upstream ion bursts of magnetospheric origin are often accompanied by relativistic $(\geq 220 \mathrm{keV})$ electrons occurring during times of active $\left(K_{p} \geq 3\right)$ geomagnetic periods (e.g. Anagnostopoulos et al., 1998, and references therein). The occurrence rate of ion and electron bursts is increased when both the solar wind

Published by Copernicus Publications on behalf of the European Geosciences Union. 


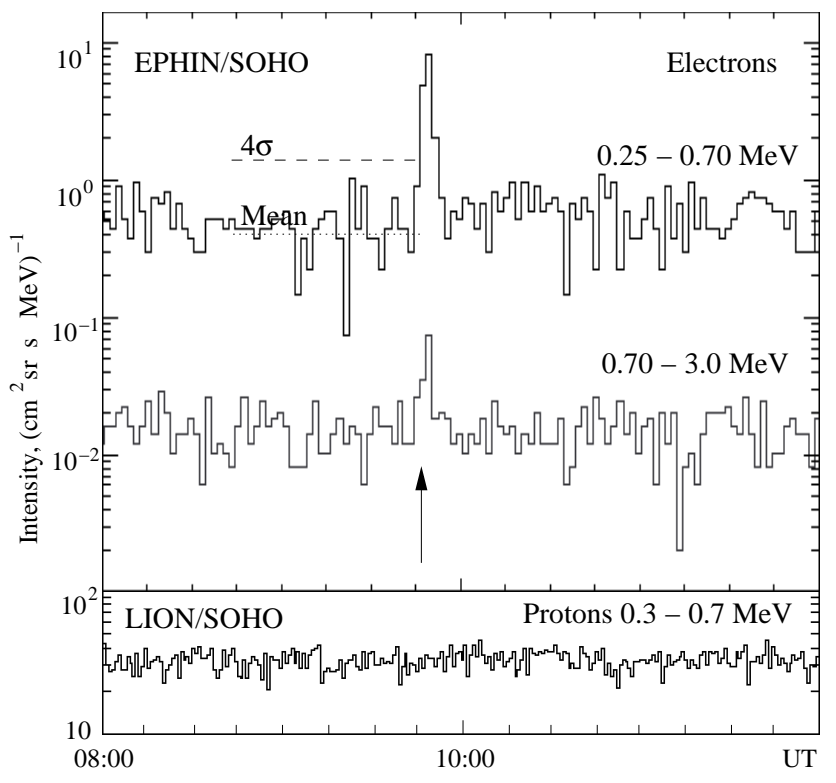

Fig. 1. Time profiles of an electron upstream event on 19 March 1996 (top, arrow) without associated proton burst (bottom). The burst is clearly detected in two energy channels (0.25-0.7 and 0.73.0 MeV). In both channels the intensities increase and decrease abruptly and nearly simultaneously. The dashed line shows the $4 \sigma$ level above $1 \mathrm{~h}$ mean value (dotted line).

speed and the geomagnetic activity index are enhanced (Desai et al., 2000; Posner et al., 2002; Klassen et al., 2006).

Most of the observations studied in the literature have been made relatively close to the bow-shock. With SOHO it is possible to extend these observations to distances far away from the bow-shock. In this paper we present a statistical analysis of the duration, the spatial distribution, the geomagnetic activity and solar wind association of energetic ( $\geq 0.25 \mathrm{MeV}$ ) electrons during 124 upstream bursts observed with the EPHIN/SOHO instrument from 1996 through 2005. Using long term observations we show for the first time distinct seasonal variations with maxima around equinoxes and minima near solstices in the occurrence number of upstream electron events.

\section{Instrumentation and data selection}

The energetic electron upstream bursts presented in this paper were observed with the Comprehensive Suprathermal and Energetic Particle Analyser (COSTEP) (Müller-Mellin et al., 1995) aboard the Solar and Heliospheric Observatory (SOHO). COSTEP comprises two instruments LION (Low Energy Ion and Electron Instrument) and EPHIN (Electron Proton Helium Instrument). LION consist of two sensor heads, each containing a double telescope, which together provide the capability to measure particle spectra in the range
$0.044-6 \mathrm{MeV}$ for protons and $0.044-0.3 \mathrm{MeV}$ for electrons. The EPHIN sensor is a multi-element array of solid state detectors with active anticoincidence to measure energy spectra of electrons in the range $0.25 \mathrm{MeV}$ to $10.4 \mathrm{MeV}$ and hydrogen and helium isotopes in the range $4 \mathrm{Mev} / \mathrm{n}$ to $>53 \mathrm{Mev} / \mathrm{n}$. For electrons there are three energy channels: $0.25-0.7,0.7-$ 3.0 and $2.6-10.4 \mathrm{MeV}$. The COSTEP aperture points along the nominal interplanetary magnetic field (IMF) at $1 \mathrm{AU}$, $45^{\circ}$ west of the spacecraft Sun line in the sunward direction. The EPHIN field of view is $83^{\circ}$ with a geometric factor of $5.1 \mathrm{~cm}^{2}$ sr. But note, after 8 July 2003 , due to a problem with the high gain antenna of SOHO, the sensor aperture points either perpendicular or along the IMF, switching every 3 months. The accumulation time of the instrument is $60 \mathrm{~s}$. Because there are no IMF measurements at SOHO we use magnetic field parameters obtained by the MFI/Wind instrument (Lepping et al., 1995). The solar wind parameters were obtained by the SOHO/CELIAS/MTOF/PM instrument (Hovestadt et al., 1995). About half of the events were also observed with the 3-D plasma instrument on board the Wind spacecraft (Lin et al., 1995) giving important confirmation that the observed electrons are really streaming from the Earth's direction.

For our study, data from 10 years of observations (January 1996 through December 2005) were used, but only events with the following criteria were selected: a) a burst was declared as significant when its intensity peak exceeded the one hour mean pre-event background by at least $4 \sigma$ and its duration over this $4 \sigma$ threshold was $\geq 2$ min (i.e. two accumulation intervals, see Fig. 1), b) the bursts were not associated with solar activity, i.e. no solar flares, type III radio bursts etc., c) when the burst was also detected by 3DP/Wind within a certain time interval $( \pm 20 \mathrm{~min})$ the electrons should arrive from the anti-Sun direction. Using these criteria we identified 124 upstream electron events during almost the whole solar cycle 23. Figure 1 shows an example of such a burst observed on 19 March 1996. The two panels display the intensity time profiles of $0.25-0.7 \mathrm{MeV}$ and $0.7-3.0 \mathrm{MeV}$ electrons and $0.3-0.7 \mathrm{MeV}$ protons as measured by the EPHIN and LION experiments, respectively. The time profiles of the electrons show abrupt rising and trailing edges at 09:50 UT and 09:55 UT. During that time no proton enhancement was observed at the spacecraft.

However some of the electron upstream events were accompanied by energetic upstream protons $(<1 \mathrm{MeV})$ as observed by the 3DP/Wind and the EPAM/ACE (Gold et al., 1998 ) instruments. D. Haggerty (private communication) argues the electron intensity increases, observed at ACE, are caused by a cross-talk of low energy protons into the electron channels. Such an effect is unlikely for the EPHIN telescope, since the aperture of the instrument is covered by two thin foils which stop protons with energies less than $0.7 \mathrm{MeV}$. Additionally our Monte Carlo simulations using GEANT code (The GEANT-4 collaboration 2006) show that protons with energies $\leq 1 \mathrm{MeV}$ are not able to produce any contamination 


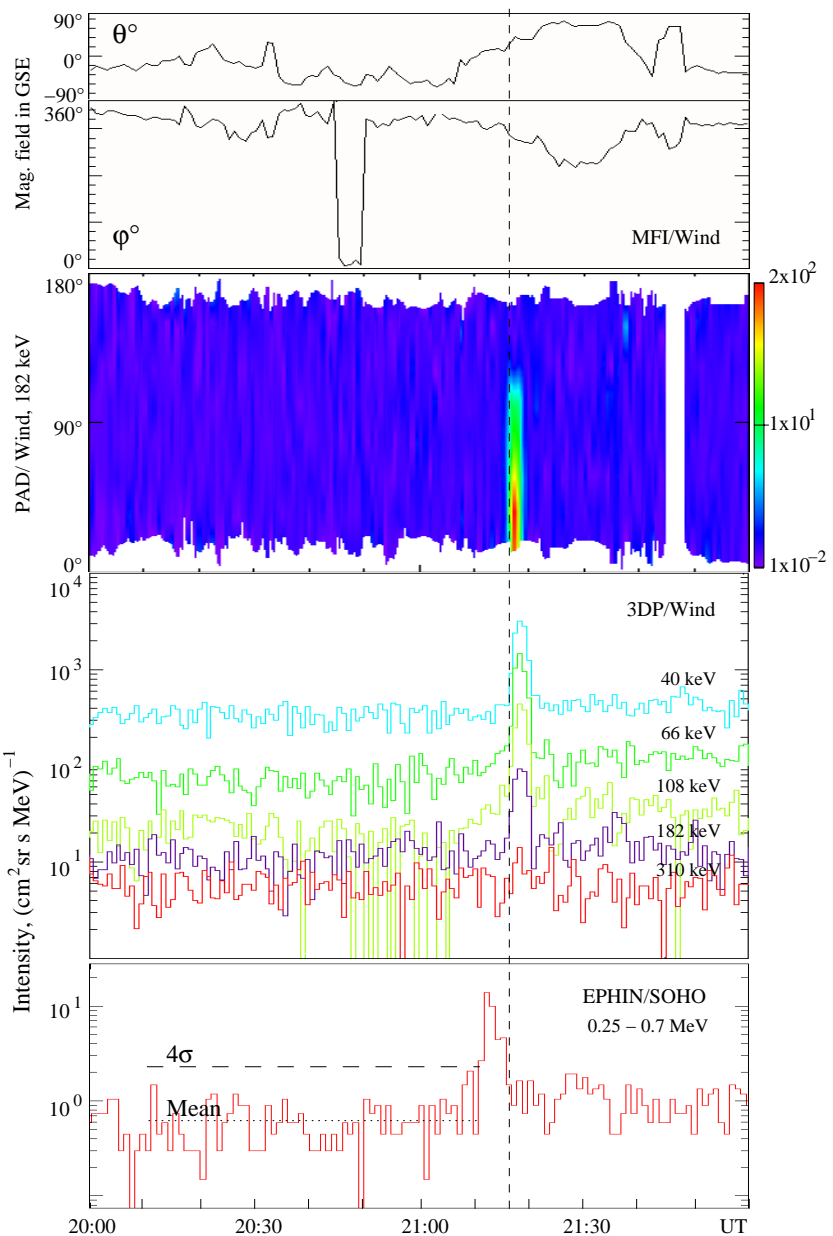

Fig. 2. Electron event on 4 April 2005 as observed with EPHIN/SOHO and 3DP/Wind near L1 (SOHO position: $\mathrm{X}_{\mathrm{GSE}}=254 R_{E} ; \mathrm{Y}_{\mathrm{GSE}}=-59 R_{E}$, Wind: $\mathrm{X}_{\mathrm{GSE}}=200 R_{E}$; $\mathrm{Y}_{\mathrm{GSE}}=-42 R_{E}$ ). Top panel shows variation of IMF components (azimuthal- $\varphi$ and elevation- $\theta$ ) during the event. Second panel is the PAD of the 3DP/Wind $182 \mathrm{keV}$ electrons. Third panel shows intensity profiles of 3DP/Wind. Bottom panel is an intensity profile of EPHIN in the energy range $0.25-0.7 \mathrm{MeV}$. The vertical dashed line indicates the burst onset time at 3DP. There is a distinct delay of 5 min between EPHIN and 3DP burst onset.

in the electron channels. Furthermore the lower panel of Fig. 1 clearly proves that no $<0.7 \mathrm{MeV}$ protons could cause the electron burst on 19 March 1996.

\section{Observations}

\subsection{Observations of individual bursts}

Before we start with observations of individual bursts, we describe the general characteristics of upstream electron events. Usually the bursts show time profiles with abrupt rising and trailing edges as shown in Fig. 1. Such profiles are very different to time profiles of solar electron events, which are

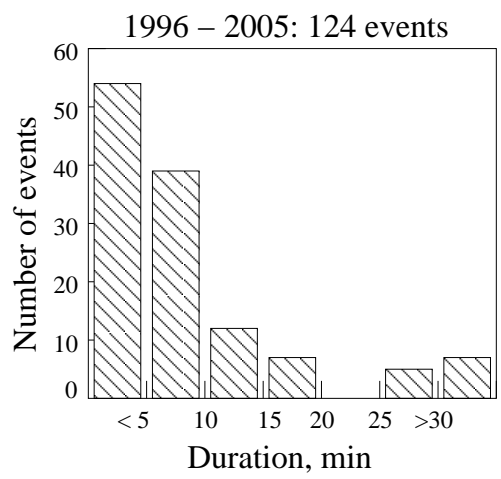

Fig. 3. Distribution of the upstream burst duration times of 124 events.

characterised by non-symmetric time profiles. The majority of upstream events are only detected in the lowest EPHIN energy channel $(0.25-0.7 \mathrm{MeV})$ and their intensities are below $20\left(\mathrm{~cm}^{2} \mathrm{sr} \mathrm{s} \mathrm{MeV}\right)^{-1}$. But $6 \%$ of the events ( 7 bursts) are also detected at higher energies of $0.7-3.0 \mathrm{MeV}$ (see Fig. 1). This burst has a nearly triangular pulse shape, lasts only $5 \mathrm{~min}$ and is not associated with protons.

Figure 2 shows an example of an electron event observed on 4 April 2005 detected with both the EPHIN aboard SOHO and the 3DP instrument aboard the Wind spacecraft. The top panels present the variation of IMF components during the event and the Pitch Angle Distribution (PAD) for $182 \mathrm{keV}$ electrons. The PAD shows a strong flux anisotropy with intensity maximum at pitch angle around $0^{\circ}$, indicating a stream away from the Earth's magnetosphere in the sunward direction. The time profiles at different energies are very similar showing a pulse character-strong sharp increase and abrupt decay profile (square/triangular pulse). There is a distinct delay of $5 \mathrm{~min}$ between SOHO/EPHIN and Wind/3DP burst onset. For this event the EPHIN aperture points along the nominal IMF in the Sun direction. It means that EPHIN detected mainly scattered electrons, because the particles streamed from the Earth's direction as observed by 3DP/Wind.

\subsection{Event durations and spatial distribution}

Figure 3 shows the distribution of duration times for all 124 events. The duration time for each individual event is measured at $4 \sigma$ level (see Figs. 1 and 2). Most events show a spiky character and last less than $10 \mathrm{~min}$ (mean $10.3 \mathrm{~min}$ ) and only about $10 \%$ last longer than $20 \mathrm{~min}$. This mean value is substantially different from the mean value of the upstream ion event duration times of $84 \mathrm{~min}$ as reported by Desai et al. (2000).

From 124 events observed by EPHIN about half (60 or $48 \%$ ) were also detected by the 3DP/Wind instrument. Because $0.25 \mathrm{MeV}$ electrons travel with more than $0.7 \cdot c$ we would expect the electron burst to occur simultaneously at 

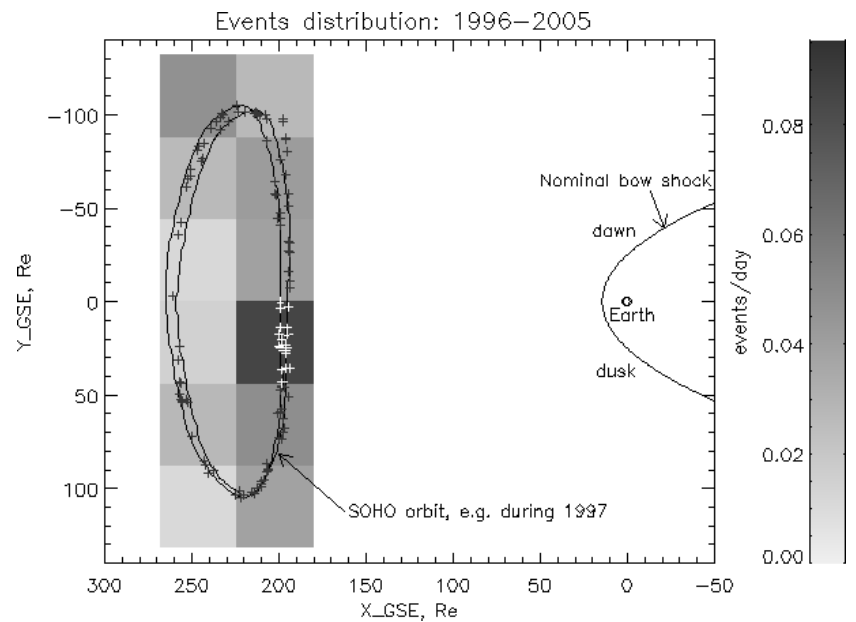

Fig. 4. Spatial occurrence rate of upstream events normalized to the number of observation days projected in the $X Y_{\mathrm{GSE}}$ plane. The darkest bins correspond to the highest occurrence rate of events. Each event is indicated by a cross.

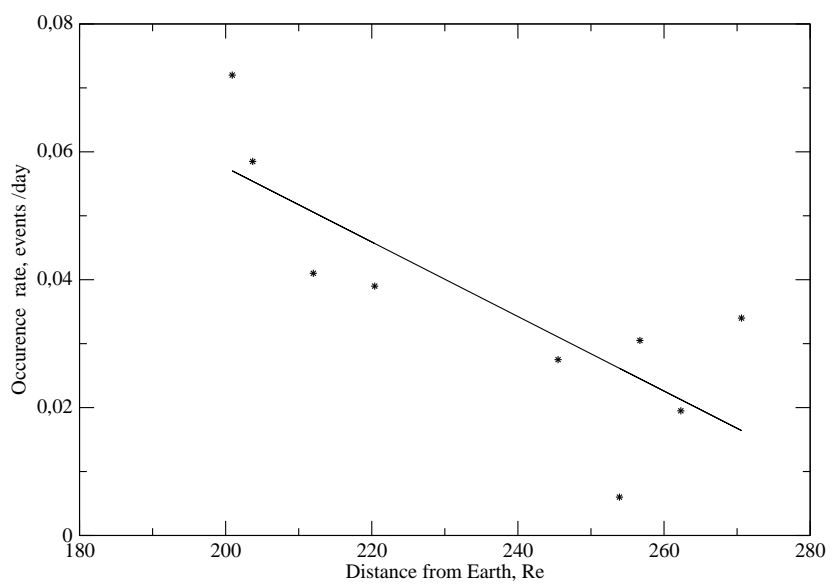

Fig. 5. Scatter plot of occurrence rate of upstream events versus distance of the spacecraft from the Earth. The line shows the linear regression fit with a correlation coefficient $r=-0.80$.

both satellites, although Wind is during most times closer to the Earth than SOHO. This is the case for only 18 of the 60 events, when the time delay was not greater than $\pm 2 \mathrm{~min}$. The remaining events have a time delay between EPHIN and 3DP onset times up to \pm 44 min (modulus mean $=9.4 \mathrm{~min}$ ), i.e. some events were first observed by EPHIN and then by 3DP $(55 \%)$ and vice versa $(45 \%)$. Such a big delay suggests that probably most events observed by SOHO and Wind were different events and they originate in different sources.

Figure 4 shows the spatial distribution and the occurrence rate of all events along the SOHO orbit. The spatial occurrence rate was obtained by dividing the number of events detected in each $44 \times 44 R_{e}$ bin by the number of observation days in that region. Darkest regions correspond to the high-

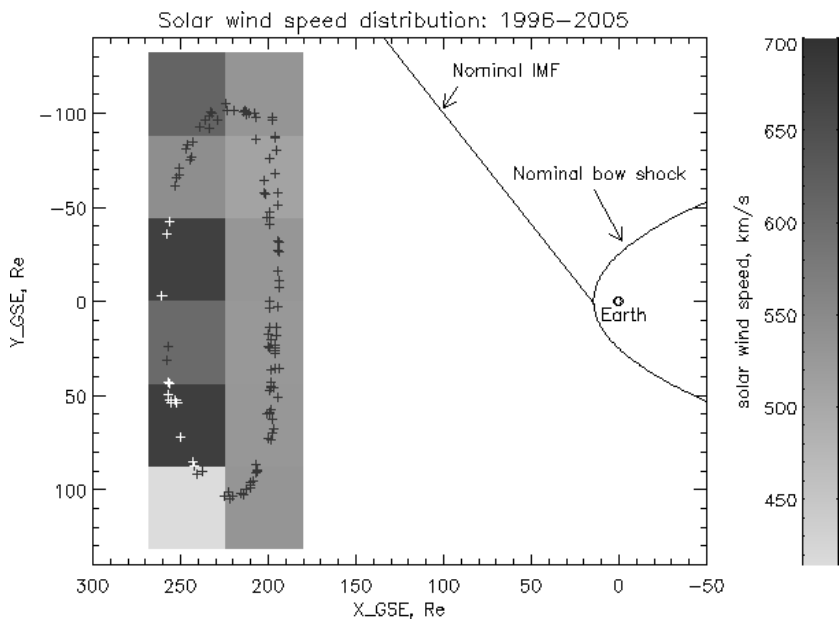

Fig. 6. Distribution of the averaged $V_{s w}$ during upstream events. The mean $V_{s w}\left(606 \mathrm{~km} \mathrm{~s}^{-1}\right)$ is higher for events detected at the remote SOHO half orbit than $V_{S w}$ at closer half orbit $\left(526 \mathrm{~km} \mathrm{~s}^{-1}\right)$.

est occurrence rate of events. The electrons were observed at all locations of the SOHO spacecraft within $190-260 R_{E}$ in $X_{\mathrm{GSE}}$ and $\pm 103 R_{E}$ in $Y_{\mathrm{GSE}}$. Most events ( 85 or $69 \%$ from all 124 events) occur when SOHO was at the half of its orbit located closer to Earth. To establish more precisely the dependence of the occurrence rate on the distance we calculate the radial distance from the Earth for each bin in Fig. 4. The occurrence rate for the equidistance bins were averaged. Although the variation in the distance for SOHO's orbit is relatively small (from 200 to $270 R_{E}$ ) there is a strong tendency with correlation coefficient of $r=-0.80$ in occurrence rate versus the distance. Namely, the event occurrence rate decreases with increasing distance from the Earth (Fig. 5).

Otherwise the occurrence rate in the dusk $\left(Y_{\mathrm{GSE}}>0\right)$ and the dawn $\left(Y_{\mathrm{GSE}}<0\right)$ sector is about the same, 0.036 and 0.032 events per day, respectively.

\subsection{Relation to geoactivity and solar wind speed}

In comparision with Fig. 4, Fig. 6 displays the distribution of solar wind speed $V_{s w}$ measured by CELIAS/SOHO during the upstream electron events. Obviously the distribution along the SOHO orbit is not uniform. For events detected at the remote half of the orbit the averaged solar wind speed of $V_{s w}=606 \mathrm{~km} \mathrm{~s}^{-1}$ is higher than at the closer one of $V_{s w}=526 \mathrm{~km} \mathrm{~s}^{-1}$. Such a distribution is expected, because in order to be connected to the magnetosphere at the remote part of the orbit the interplanetary magnetic field has to be more radial. Thus higher solar wind speeds should be observed.

Figure 7 (left) shows the distribution of the mean monthly $A_{p}$ index in comparison with the monthly number of upstream events during 1996-2005. Both the $A_{p}$ index and the number of upstream events show a semiannual (seasonal) variation with maxima around equinoxes and minima near 

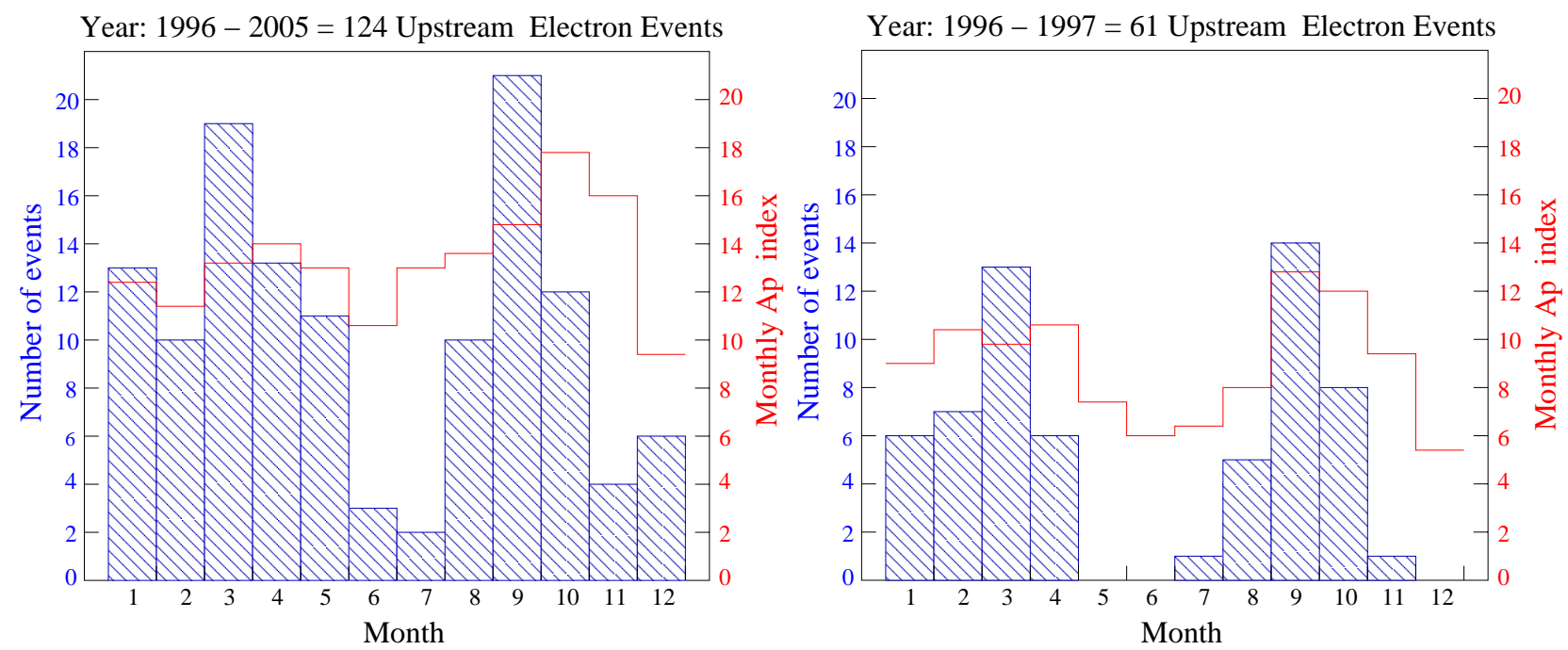

Fig. 7. The seasonal variation of the occurrence of upstream events in comparison with the $A_{p}$ index during solar cycle 23 (1996-2005) and during solar minimum (1996-1997). The number of upstream events shows distinct maxima around equinoxes (March-April; SeptemberOctober) and minima near solstices (June-July; November-January). This variation is more pronounced during solar activity minimum for the years 1996-1997 (right).

solstices. In general, this variation is more pronounced in the distribution of upstream events especially near the minimum of solar activity during 1996-1997, when the influences of solar flares, CMEs etc. on the magnetosphere were weak (Fig. 7, right). Note that the minimum in summer (June-July) is deeper than in winter (December-January).

Figure 8 presents the results of a superposed epoch analysis. On the left the distribution of the daily $A_{p}$ index and on the right the distribution of the daily mean solar wind speed $\left(V_{s w}\right)$ are shown around the day of the upstream events. Day zero corresponds to the day of the upstream event. The figure suggests that the probability to observe an upstream event is higher one day after the maximum of the $A_{p}$ index and during the maximum of solar wind speeds $V_{s w}$. By day " 0 " the mean $V_{s w}=507 \mathrm{~km} \mathrm{~s}^{-1}$.

In comparision to Fig. 8, Fig. 9 displays the distribution of the IMF components $B_{y}$ (left) and $B_{z}$ (right) around the days of upstream events. While the distribution of the $B_{y}$ component does not show significant maxima, the $B_{z}$ component is southwards directed and has a strong maximum at day " 0 " like the distribution of the $A_{p}$ index and of the solar wind speed $V_{s w}$.

\subsection{Observational summary}

We have analyzed the statistical parameters of 124 electron upstream events in the energy range $0.25-3.0 \mathrm{MeV}$ observed by the EPHIN/SOHO instrument from 1996 to 2005 and their associations with geomagnetic and interplanetary conditions. The main observational results are the following:
1. The majority of events were detected in the energy range $0.25-0.7 \mathrm{MeV}$, but a small fraction of events $(6 \%)$ were detected also in the range $0.7-3.0 \mathrm{MeV}$.

2. Almost all bursts show nearly square/triangular pulse shaped time profiles which differ strongly from solar electron events.

3. Most events have a time duration $\leq 10 \mathrm{~min}$.

4. The event occurrence number shows a distinct seasonal variation with maxima around equinoxes and minima near solstices.

5. Superposed epoch analysis shows that on average the upstream events were observed during the same day when $V_{s w}$ and $B_{z}$ southward component reach their maxima and one day later than the maximum of $A_{p}$ index.

6. The events occurred along the whole orbit of SOHO (Fig. 4), but the event occurrence rate decreases with increasing distance of the spacecraft from the Earth. The events detected at the remote half orbit were associated with higher solar wind speeds than those closer to Earth.

\section{Discussion}

The EPHIN/SOHO observations of upstream electron events far from the Earth's bow-shock near the L1 point show that electron bursts appear during enhanced conditions of solar wind speed and geomagnetic activity. In previous studies it 


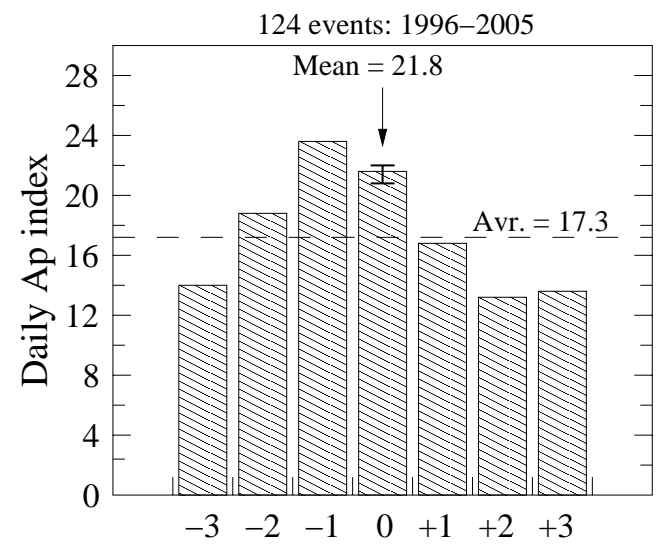

Days before and after event

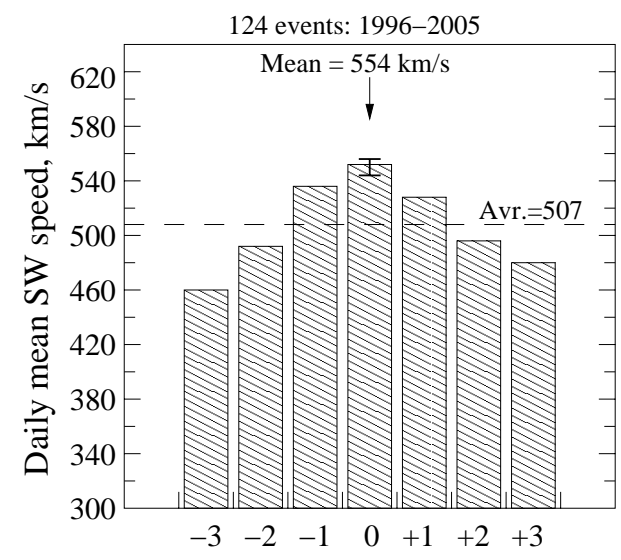

Days before and after event

Fig. 8. Superposed epoch analysis. Daily $A_{p}$ index and daily mean solar wind speed $V_{s w}$ around the upstream event onset. Day zero corresponds to the day of the upstream event.

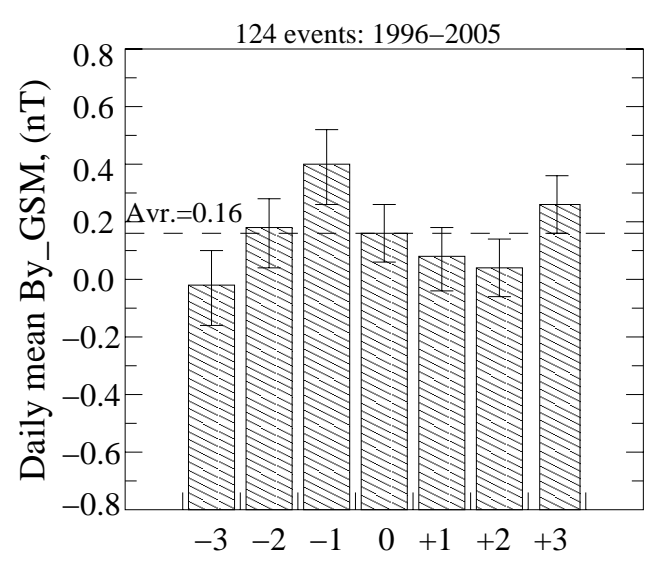

Days before and after event

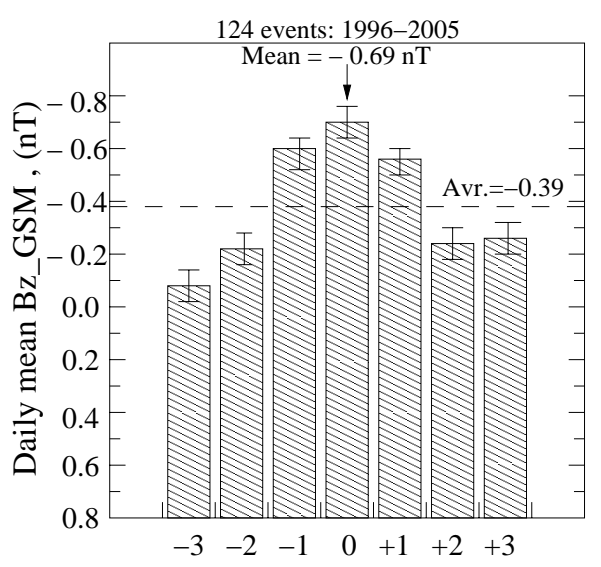

Days before and after event

Fig. 9. Daily $B_{y}(\mathrm{GSM})$ and $B_{z}(\mathrm{GSM})$ components around the upstream event onset.

was found that the occurrence rate of proton upstream events is correlated with solar wind speed $V_{s w}$ and $K_{p}$ index (e.g. Anagnostopoulos et al., 1998, 1999; Desai et al., 2000). Our results confirm these findings and show in addition that the distribution of electron events has a good correlation with the $B_{z}$ southward component (see Fig. 9). Most of the events occur near the maxima of the $A_{p}$ index, the solar wind speed $V_{s w}$ and the $B_{z}$ southward component. The positive correlation between $V_{s w}$, geomagnetic activity and upstream event rate has been used as evidence in support of magnetospheric leakage model (e.g. Anagnostopoulos et al., 1999).

Our finding of a strong semiannual variation of the number of electron events (Fig. 7) also supports the model that these bursts are rather of magnetospheric origin than bow-shock associated as discussed by Krimigis et al. (1978) and Scholer et al. (1981). Furthermore a most of magnetospheric parameters show similar seasonal variations, e.g. aurora occurrence, $K_{p}$ and $A_{p}$ indices, etc.

Seasonal variation and a strong maximum in $B_{z}, A_{p}$ and $V_{s w}$ (Figs. 7-9) show the relevance of the Russell-McPherron mechanism (Russell and McPherron, 1973) for occurrence of upstream events. This mechanism is based on the reconnection model of IMF and geomagnetic field lines, and takes into account the southward component of the IMF relative to the geomagnetic field direction. This mechanism predicts maximal southward $B_{z}$ component in GSM coordinates and a maximum in geomagnetic activity in early April and October.

The magnetospheric origin of observed electrons is also supported by SAMPEX and STICS/Wind observations of a 
similar semiannual variation of $\mathrm{MeV}$ electrons in the outer radiation belt (Fig. 3 in $\mathrm{Li}$ et al., 2001) and of heavy ions upstream of the bow-shock (Posner et al., 2002).

On the other hand we can not totally exclude the model of electron acceleration at the bow-shock, because the higher $V_{s w}$ results in a stronger compression at the bow-shock which can lead to increased efficiency of the bow-shock acceleration (e.g. Burgess, 2007). Therefore the Fermi acceleration process or the drift acceleration mechanism are alternative processes which can explain the generation of upstream electron events (e.g. Wu, 1984; Krauss-Varban and Wu, 1989; Burgess, 2006, 2007). However, we note that to our knowledge, there is nothing in the literature reporting that the bow-shock acceleration mechanisms can act in seasonal regime and that they can accelerate electrons above 0.25$0.70 \mathrm{MeV}$ (e.g. Anagnastopoulos et al., 1998, 2005, Burgess, 2007, and references therein).

Desai et al. (2000) and Posner et al. (2002) found that the majority of ion events occur in the dawn-noon sector under a radial IMF. Otherwise the greater intensities of ion events are observed at the dusk sector (Anagnostopoulos et al., 2005). We found that this is not the case for electron events. Generally the spatial distribution of electron events in the dusk and dawn sectors is more or less uniform (Fig. 4). Thus, 53\% (47\%) of events occur in the dusk (dawn) sector, respectively. Such uniform distribution of electron events indicates that the majority of electrons could escape from the dusk as well as from the dawn sector. This establishes a problem for both the leakage model and for the bow-shock acceleration models (Sarris et al., 1978; Anderson, 1981). Because both models predict a dusk-dawn asymmetry in spatial distribution of particles upstream of the bow-shock (e.g. Anagnostopoulos et al., 2005).

Scholer et al. (1981) suggested that accelerated magnetospheric particles may leak out of the magnetosphere at certain locations but are not necessarily distributed over the whole environment of the magnetosphere. If these electrons were released from the whole magnetosphere we would expect that they reach Wind and SOHO with a short time delay. Such a simultaneous occurrence (within $\pm 2 \mathrm{~min}$ ) has been observed during only 18 electron events when the separation between the spacecraft was between 50 and $290 R_{E}$. Since both spacecraft were probably connected to different parts of the magnetosphere the source of these electrons may have been distributed over a substantial part of the magnetosphere with the source size being comparable to the bow-shock (see also Haggerty et al., 2000).

The formation of observed square/triangular time profiles depends on

1. the magnetic connection of the satellite to the source,

2. the propagation conditions, and

3. the injection profile at the source.
The time profiles of almost all bursts exhibit nearly square/triangular pulse shapes with abrupt rising and trailing edges. Unfortunately the COSTEP/SOHO instrument does not provide information about the pitch angle distribution of the electron events. The 3DP experiment on the spinning satellite Wind, however, provides these directionality information. The detected bursts are highly anisotropic (Fig. 2). Futhermore, the magnetic field as observed by Wind does not show prompt variations during most of the events. This implies that the magnetic connection from the spacecraft to the Earth magnetosphere was not altered during the whole event. This is therefore also valid for the particle propagation from the source to the spacecraft. But if these conditions are nearly constant, we have to conclude that the observed time profile reflects the injection profile (window) at the source. In this model upstream electrons escape through a "window" in the magnetosphere triggered by reconnection processes of the IMF with the geomagnetic field, which leads to the observed "square/triangular" time profiles. These "windows" occur during strong southward $B_{z}$ component, which favour these reconnection processes. It is well known that such processes can occur on short time scales leading to "deltafunction" like injection.

\section{Conclusions}

We have analysed the duration times, the spatial distribution, and the association with solar wind and geoactivity of electron upstream events observed by the EPHIN instrument on board the SOHO spacecraft at the L1 point from 1995 through 2005. It is shown that some upstream events are detected at energies above $0.7 \mathrm{MeV}$. We found that the occurrence number of upstream electron events shows distinct seasonal variations with maxima around equinoxes and minima near solstices. The occurrence of electron bursts is correlated with maxima in $V_{s w}$, in $A_{p}$ index and with southward interplanetary magnetic field component $\left(B_{z}\right)$. We consider that the observed correlations and the seasonal variations are resonable arguments to favour the magnetospheric leakage mechanism to explain the occurrence of electron upstream events.

Acknowledgements. We thank the 3DP/Wind team for data access and D. Haggerty for discussion about possible contamination of electron channels by protons. We acknowledge a useful discussion with B. Klecker. This work was supported by DLR under grant number 50 OC 0105 and by the Junta de Comunidades de CastillaLa Mancha and the European Social Fund.

Topical Editor I. A. Daglis thanks two anonymous referees for their help in evaluating this paper. 


\section{References}

Anagnostopoulos, G. C., Rigas, A. G., and Sarris, E. T.: Characteristics of upstream energetic $(\mathrm{E}>50 \mathrm{keV})$ ion events during intense geomagnetic activity, J. Geophys. Res., 103, 9521-9533, 1998.

Anagnostopoulos, G. C., Kaliabetsos, G., Argyropoulos, G., and Sarris, E. T.: High energy ions and electrons upstream from the earth's bow shock and their dependence on geomagnetic conditions: Statistical results between years 1982-1988, Geophys. Res. Lett., 26, 2151-2154, 1999.

Anagnostopoulos, G. C., Vassiliadis, E. S., Karanikola, I.: Dawndusk asymmetry in spatial distribution and origin of energetic ion events upstream the Earth's bow shock, Planet. Space Sci., 53, 53-58, 2005.

Anderson, K. A.: Measurements of bow shock particles far upstream from the earth, J. Geophys. Res., 86, 4445-4454, 1981.

Asbridge, J. R., Bame, S. J., and Strong, I. B.: Outward flow of protons from the Earth's bow shock, J. Geophys. Res., 73, 57775782, 1968.

Balogh, A., Hynds, R. J., van Rooijen, J. J., Stevens, G., Domingo, V., Sanderson, T. R., and Wenzel, K.-P.: Evidence for magnetosphere proton bursts: First results from the low energy proton experiment on ISEE 3 (abstract), Eos Trans. AGU, 59, 1174, 1978.

Bieber, J. W. and Stone, E. C.: Energetic electrons in the magnetosheath and upstream of the bow shock, J. Geophys. Res., 87, 85-94, 1982.

Burgess, D.: Simulation of electron acceleration at collisionless shocks: The effects of surface fluctuations, Astrophys. J., 653, 316-324, 2006.

Burgess, D.: Particle acceleration at the Earth's bow shock, Lect. Notes Phys., 725, 161-190, 2007.

Desai, M. I., Mason, G. M., Dwyer, J. R., Mazur, J. E., von Rosenvinge, T. T., and Lepping, R. P.: Characteristics of energetic ( $\geq 30 \mathrm{keV} /$ nucleon) ions observed by the Wind/STEP instrument upstream of the Earth's bow shock, J. Geophys. Res., 105, 61-78, 2000.

Fan, C. Y., Gloeckler, G, and Simpson, J. A.: Evidence for $>30 \mathrm{keV}$ electrons accelerated in the shock transition region beyond the earth's magnetospheric boundary, Phys. Rev. Lett., 13, 149-152, 1964.

Formisano, V.: Properties of energetic electrons of magnetospheric origin in the magnetosheath and in the solar wind-Correlation with geomagnetic activity, Planet. Space Sci., 27, 867-879, 1979.

Gold, R. E., Krimigis, S.M., Hawkins, S. E., Haggerty, D. K., Lohr, D. A., Fiore, E., Armstrong, T. P., Holland, G., and Lanzerotti, L. J.: Electron, Proton, and Alpha Monitor on the Advanced Composition Explorer spacecraft, Space Sci. Rev., 86, 541-562, 1998.

Haggerty, D. K., Roelof, E. C., Smith, C. W., Ness, N. F., Tokar, R. L., and Skoug, R. M.: Interplanetary magnetic field connection to the L1 Lagrangian orbit during upstream energetic ion events, J. Geophys. Res., 105, 25 123-25 131, 2000.
Hovestadt, D., Hilchenbach, M., Bürgi, A., et al.: CELIAS-Charge, element and isotope analysis system for SOHO, Sol. Phys., 162, 441-481, 1995.

Klassen, A., Gómez-Herrero, R., Böhm, E., Müller-Mellin, R., Heber, B., and Wimmer-Schweingruber, R. F.: COSTEP/SOHO observations of energetic electrons far upstream of the Earth's bow-shock, Proceedings of SOHO-17, ESA SP-617, 2006.

Krauss-Varban, D. and Wu, C. S.: Fast Fermi and gradient drift acceleration of electrons at nearly perpendicular collisionless shocks, J. Geophys. Res., 94, 15 367-15 372, 1989.

Krimigis, S. M., Venkatesan, D., Barichello, J. C., and Sarris, E. T.: Simultaneous measurements of energetic protons and electrons in the distant magnetosheath, magnetotail, and upstream in the solar wind, Geophys. Res. Lett., 5, 961-964, 1978.

Lepping, R. P., Acuna, M. H., Burlaga, L. F., et al.: The Wind Magnetic Field Investigation, Space Sci. Rev., 71, 207-229, 1995.

Li, X., Baker, D. N., Kanekal, S. G., Looper, M., and Temerin, M.: Long term measurements of radiation belts by SAMPEX and their variations, Geophys. Res. Lett., 28, 3827-3830, 2001.

Lin, R. P., Anderson, K. A., Ashford, S., et al.: A three-dimensional plasma and energetic particle investigation for the Wind spacecraft, Space Sci. Rev., 71, 125-153, 1995.

Müller-Mellin, R., Kunow, H., Fleissner, V., et al.: COSTEP comprehensive suprathermal and energetic particle analyser, Sol. Phys., 162, 483-504, 1995.

Posner, A., Schwadron, N. A., Zurbuchen, T. H., Kozyra, U., Liemohn, M. W., and Gloeckler, G.: Association of low-chargestate heavy ions up to $200 \mathrm{Re}$ upstream of the Earth's bow shock with geomagnetic disturbances, Geophys. Res. Lett., 29, 3-1, doi:10.1029/2001GLO13449, 2002.

Russell, C. T. and McPherron, R. L.: Semiannual Variation of Geomagnetic Activity, J. Geophys. Res., 78(1), 92-108, 1973.

Sanderson, T. R., Reinhard, R., and Wenzel, K.-P.: The propagation of upstream protons between the Earth's bow shock and ISEE 3, J. Geophys. Res., 86, 4425-4434, 1981.

Sarris, E. T., Krimigis, S. M., Bostrom, C. O., and Armstrong, T. P.: Simultaneous multispacecraft observations of energetic proton bursts inside and outside the magnetosphere, J. Geophys. Res., 83, 4289-4305, 1978.

Scholer, M., Hovestadt, D., Ipavich, F. M., and Gloeckler, G.: Upstream energetic ions and electrons: Bow shock-associated or magnetospheric origin?, J. Geophys. Res., 86, 9040-9046, 1981.

The GEANT4 collaboration.: An object-oriented toolkit for simulation in HEP, CERN-LHCC 98-44, 2006.

Wu, C. S.: A fast Fermi process - Energetic electrons accelerated by a nearly perpendicular bow shock, J. Geophys., Res., 89, 8857$8862,1984$. 